\title{
PARADEROS COMPLEMENTARIOS PARA EL TRANSPORTE PÚBLICO EN ESPACIOS REDUCIDOS DE LA ZONA CÉNTRICA EN LA CIUDAD DE SAN JUAN DE PASTO
}

\section{Entorno Ciudad}

Erika Vanessa Muñoz Cuarán

evmunozc@gmail.com

Kevin Alexander Montenegro Bravo

Universidad de Nariño

Grupo CORD

Pasto, Nariño. 


\section{Resumen}

a ciudad de San Juan de Pasto (Colombia) continúa creciendo y con ello presenta varios - cambios. En este caso, se hace referencia a los cambios en infraestructura y movilidad, sistema circulatorio indispensable para la productividad y mejora de la calidad de vida de los habitantes. Por su parte, la movilidad viene siendo afectada debido al incremento del parque vehicular y el uso de transporte informal; de ahí que el servicio de transporte público se ha visto afectado por un decrecimiento en el número de pasajeros, pese a los esfuerzos realizados por el Sistema Estratégico de Transporte Público de pasajeros de la ciudad de Pasto (Avante, 2002), el cual ha incorporado políticas regulatorias a través de las cuales se promueve el transporte público con un sistema de paraderos que busca organizar el ascenso y descenso de los pasajeros de los vehículos e información de los recorridos; pero, lastimosamente, con débiles resultados sobre la movilidad, ya que no tuvo el impacto esperado sobre los pasajeros y los conductores. Por este motivo, la investigación buscó encontrar las causas y una solución desde el Diseño industrial, para que se organice un adecuado servicio de transporte público. Para esto, se desarrolló una estrategia metodológica desde la investigación- creación, para determinar una solución de complejidad media que contribuya a generar una movilidad sostenible; también se conformó un focus group, con una muestra de 20 personas. Se obtuvo como resultado un $60 \%$ de aspectos negativos, entre ellos la falta de divulgación, falencias en la usabilidad, no presentan inclusión social y falta de visibilidad; y un $40 \%$ en aspectos positivos, como la importancia de la información de rutas, cultura ciudadana, efectividad y protección. Se llegó a la conclusión de que la adaptabilidad es clave para la elaboración del diseño que se quiere incorporar, siempre y cuando se cumpla con los requerimientos necesarios para formar un diseño adaptable en condiciones complejas y modulares, para poder colonizar varios espacios reducidos.

\section{Introducción}

El proyecto se origina desde una experiencia ciudadana en la ciudad de Pasto, la cual se enfocó en analizar problemas de contexto en la movilidad urbana y, así mismo, plantear soluciones de complejidad media. La ciudad está en un constante cambio ya sea cultural, económico o de infraestructura, por lo que se generan diferentes problemas para la ciudadanía, algunos de los cuales se retoman en el Plan de Ordenamiento Territorial (POT, 2015); la estructura y el modelo urbano que propone, es el de ciudad compacta, es decir, un equilibrio entre lo construido y el espacio público. No obstante, Pasto, según lo observado, carece de espacio público; esto se ve reflejado en andenes angostos en la zona central, las medidas no superando los 2 metros de ancho, en ambos costados de la vía, lo cual complica la circulación peatonal y pone en peligro a los transeúntes.

Por su parte, para la movilidad la situación actual es caótica, entre otras razones porque existe una alta tasa de motorización, estimada en 185.000 vehículos registrados en el 2018 y 258.719 registrados en el 2019 (RUNT, 2019). Según esto, de los 402.900 habitantes de Pasto (DANE, Proyección de población, 2018), 1 de cada 2, en la parte urbana, tiene vehículo. 
Por esta problemática, surge la necesidad de elaborar el Plan Maestro de Movilidad, como una ruta que permita establecer estrategias para mejorar las condiciones de vida de las personas. El Plan se estableció desde el 28 de junio del 2017, con una inversión de \$1.350.000.000, contando con tres fases de ejecución destinadas a infraestructura, sistema de gestión y control de flota, semaforización, recaudo, señalética y paraderos, patios y talleres (Avante-Sistema Estrategico de Trasnporte Público, 2017).

En la actualidad, ya se ha puesto en marcha el sistema de paraderos verticales con 700 señales en todas las comunas y la mayoría de los corregimientos del Municipio de Pasto en lo que va de 2016 a 2019, con una inversión de \$928 millones de pesos (Secretaría de Tránsito y Transporte del Municipio de Pasto, 2019). Pero, según lo observado, no se hace notoria la usabilidad de estos artefactos, ya que una mayoría de usuarios continúa abordando los buses en cualquier sitio y los conductores recogiendo pasajeros donde el usuario lo requiera.

Por esta razón, el proyecto de Laboratorio de Movilidad (MOV-LAB) del grupo CORD de la Universidad de Nariño -que nace a partir de la iniciativa de desarrollar proyectos de complejidad media en la movilidad de la ciudad de San Juan de Pasto, al identificar oportunidades de diseño que puedan llegar a impactar problemáticas especificas-; en este caso, se ha orientado a mejorar la calidad del servicio en el transporte público para los ciudadanos. Por este motivo, se ha propuesto la estrategia de paraderos complementarios de transporte público en la parte céntrica de la ciudad, bajo una metodología adecuada que ayude a identificar problemas relacionados al contexto y poder darles solución de manera efectiva, para garantizar a la ciudadanía, resultados óptimos en el servicio de transporte público. Sin duda es un reto grande, pero que vale la pena asumir, por el progreso de una ciudad amigable.

Para el estudio se recolectó la información posible con fotografías, grupo focal, referentes mundiales, referentes nacionales, matriz de comportamiento del usuario en el contexto, se detalló la diversidad de la ciudad y su estructura colonial, estudio de paraderos existentes, mapas mentales, exploración de conceptos de diseño basados en la naturaleza y la cultura.

En la investigación, la cultura ciudadana desempeña un papel importante, ya que, si bien es cierto, el pastuso tiene la costumbre de esperar el autobús en cualquier sitio, así mismo descender en la puerta de la casa si es posible, con lo que aumenta los tiempos de espera de los otros pasajeros, con lo que el servicio de transporte público comienza a fallar; costumbre que no se ha podido cambiar, ya que el mismo conductor del transporte público no tiene en cuenta los paraderos tipo 1 que ha implementado Avante; además de que estos elementos con señal vertical, se ubican en espacios en dónde el andén no supera los 2.20 metros. Aun así, se pretende implementar el segundo tipo de paraderos con mobiliario para espacios superiores a 3.80 metros, diseñados para ciudades grandes. 


\section{Propósito de la Investigación}

Los recorridos y todas las formas de desplazamiento necesitan, conforme a los objetos y motivaciones del usuario, no sólo un sistema de referencias que permita su orientación autónoma sino, también, es importante un ambiente diseñado para que el usuario pueda permanecer de manera segura, cómoda y grata en el lugar en los momentos de espera de los vehículos; reduciendo la necesidad de solicitar ayuda a otros individuos y concentrando a los usuarios en un punto definido, para así cumplir con los tiempos en rutas y el individuo tenga una interacción real con el sistema de transporte público.

De esta manera, el proyecto adquiere valor no solamente para el diseñador sino, también, para el empresario, quien deberá reconocer que este medio integrado con los objetos, es eficaz, rentable y permanente en el difícil reto de vincular a la empresa y sus usuarios de forma ordenada, lo cual contribuirá a mejorar algunos aspectos en los sistemas implantados por las organizaciones que trabajan en conjunto.

\section{Metodología}

Investigación Creación Contexto / Sensación Detonante / Materialización.

Respecto a la investigación creación, Ballesteros (2018) argumenta:

La investigación creación se caracteriza por desarrollar procesos altamente diversos, procesos influenciados por el carácter personal del creador, su contexto y lenguaje formal estético. Por lo tanto, no es prudente encajar dichos procesos en métodos estándar o rígidos que limiten en cierta manera la creatividad.

En ese sentido, no es posible afirmar que la investigación creación se rija bajo un solo orden o método, pero si deben existir ciertos requerimientos necesarios para generar soluciones desde un modelo de investigación creación. Se plantean, entonces, tres componentes de una estructura general para el proceso de investigación creación, que pueden ser secuenciales, simultáneos o en orden indistinto.

Contextualización: consiste en conocer y apropiar la realidad para alimentar los procesos de creación. Se equipará a una búsqueda de diversos tipos de información, lo cual determina la originalidad y novedad de la producción de conocimiento mediante la revisión de referentes desde su marco disciplinar.

Sensación detonante: consiste en la toma de decisiones respecto a la interpretación, reflexión y crítica respecto a la información del contexto recabada. Así, el investigador creador asume una postura frente a la realidad contextual, que le permite enfatizar en un aspecto particular de su interés. 
Conformación plástica: Consiste en la transformación de la realidad implícita en la creación, la cual adquiere una forma y lenguaje específicos, que le permiten comunicar el mensaje que contiene. En otras palabras, se concreta la creación en un producto plástico sensorial.

\section{- Mapa metodológico investigación creación de la comunicación en la movilidad urbana}

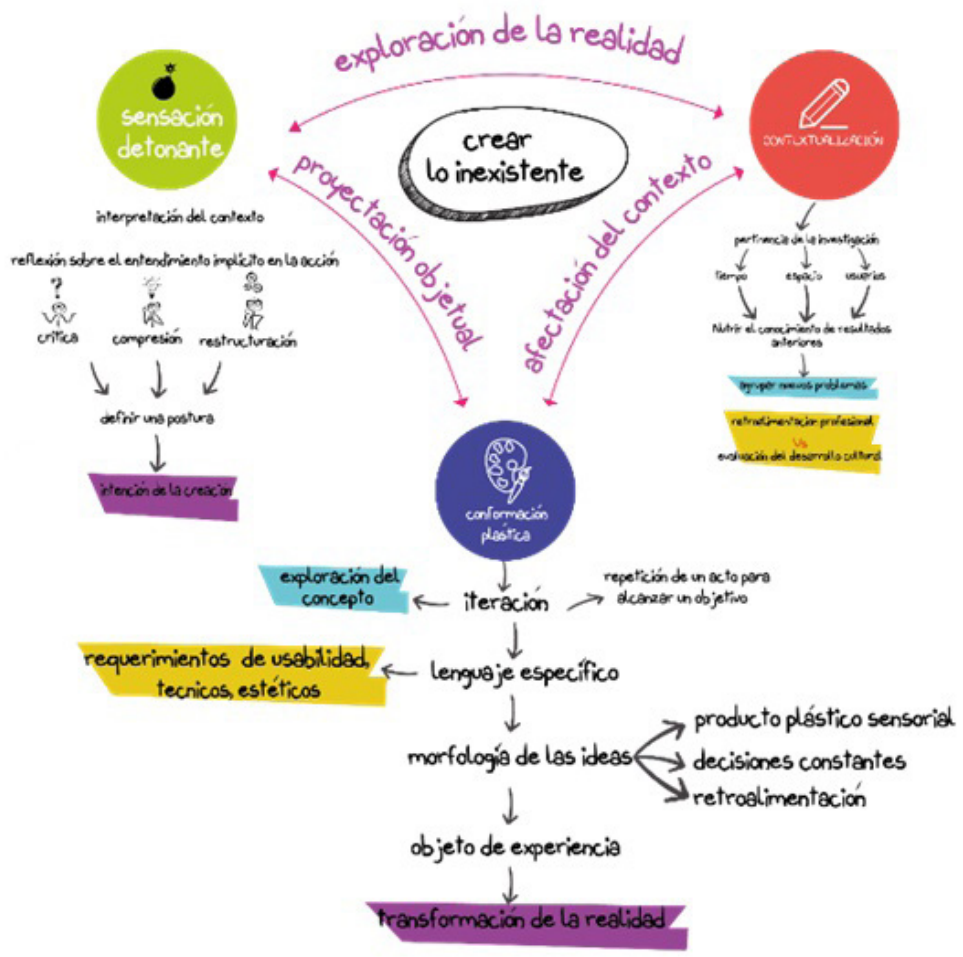

Figura 1. Construcción de la investigación

En la figura 1 se muestra la metodología y los momentos con los que se abordó la problemática que se viene presentando en la movilidad de transporte urbano y buscar las posibles soluciones, con la conceptualización de los procesos diversos que tiene una investigación, principalmente se cuenta con la exploración o contextualización del medio; en este aspecto los estímulos sensoriales juegan un papel importante en cómo se puede ver el mundo y desde qué perspectiva se aprecian los acontecimientos que pasan alrededor. Una vez que se entiende el porqué de las cosas, se pasa a la construcción de un sistema conceptual, al tomar varios puntos de vista desde otras disciplinas. Este sistema está implícito en productos o servicios que son las soluciones contra dicho problema. 


\section{- Primer momento}

Exploración de la realidad. Se trazó un mapa mental básico de los factores de investigación conocidos desde la disciplina, que estén presentes en el fenómeno de la movilidad urbana. El primer factor fue la observación, desde una perspectiva ciudadana, del panorama en el transporte público, con la cual se identificó los paraderos verticales instalados por Avante, en donde la interacción con los usuarios estaba ausente. El segundo factor fue establecer algunas causas por las cuales ocurría este tipo de omisión por parte de las personas; y, el tercer factor fue establecer algunas consecuencias que podría conllevar un mal servicio de transporte público en la ciudad de Pasto.

\section{Interpretación del contexto y el usuario}

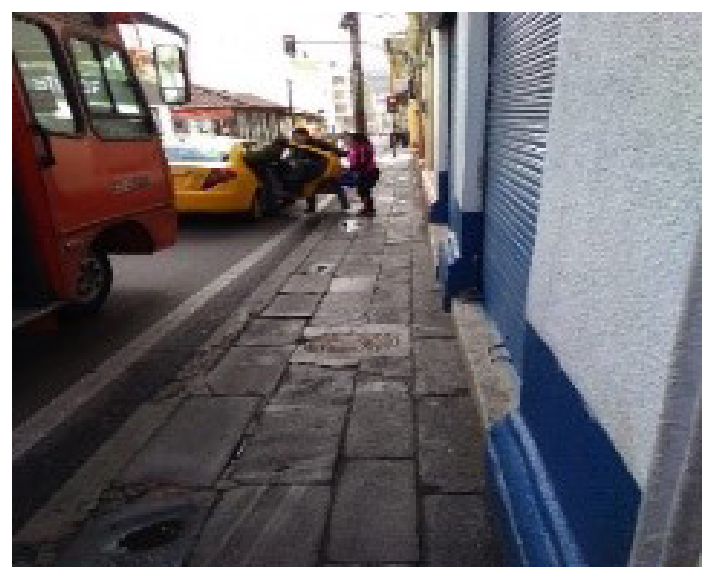

Figura 2. Ancho insuficiente de los andenes en el centro de la ciudad Fuente: Archivo fotográfico del autor

Estudio de campo. Mediante el desarrollo del estudio en Pasto, se hizo un registro fotográfico de los andenes en el centro de la ciudad, para notar que muchos de ellos no superan 1 metro de ancho entre la residencia y la calle (véase figura 2). Según el estudio realizado por la firma Duarte Guterman en el Plan de Ordenamiento Territorial de 2015, concluye que:

\footnotetext{
Los diseños geométricos de las vías, y en particular los accesos a barrios, son inadecuados, en general no disponen de andenes o son muy angostos. El $25 \%$ de la malla vial dispone de menos de 2 metros de andenes en sus dos costados; el $46 \%$ tiene andenes mayores de 2 y menores de 4 y el $30 \%$ tiene andenes mayores de 4 metros. Todo lo cual conlleva un alto potencial de accidentalidad por maniobras repentinas e improvisadas de los vehículos" (POT, 2015).
}

Por lo que se hace evidente un diseño de paradero que se acople a estas condiciones físicas y, por supuesto, los paraderos no cubrirán la mayor parte de la malla vial, precisamente, por los andenes estrechos; así, se hace necesario un elemento menos invasivo, pero que cumpla con la función de tener un servicio de transporte organizado y dinámico. De igual 
manera, se observó que tanto los pasajeros como los conductores no respetan las señales verticales existentes que indican un paradero.

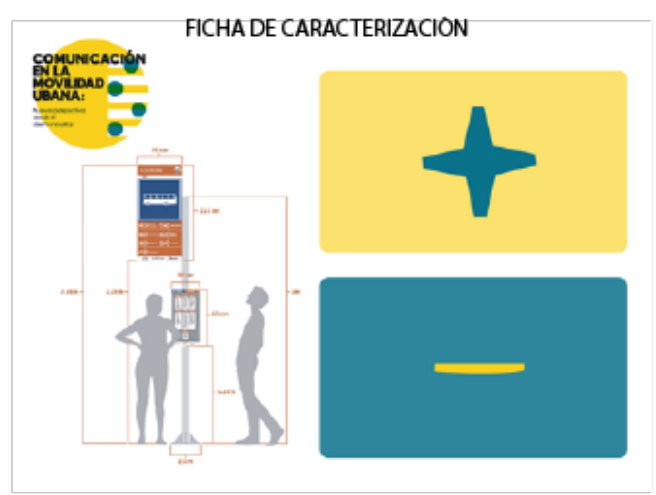

RESULTADOS OBTENIDOS

COMPONENTE POSTINO COMPONENTE NEGATNO
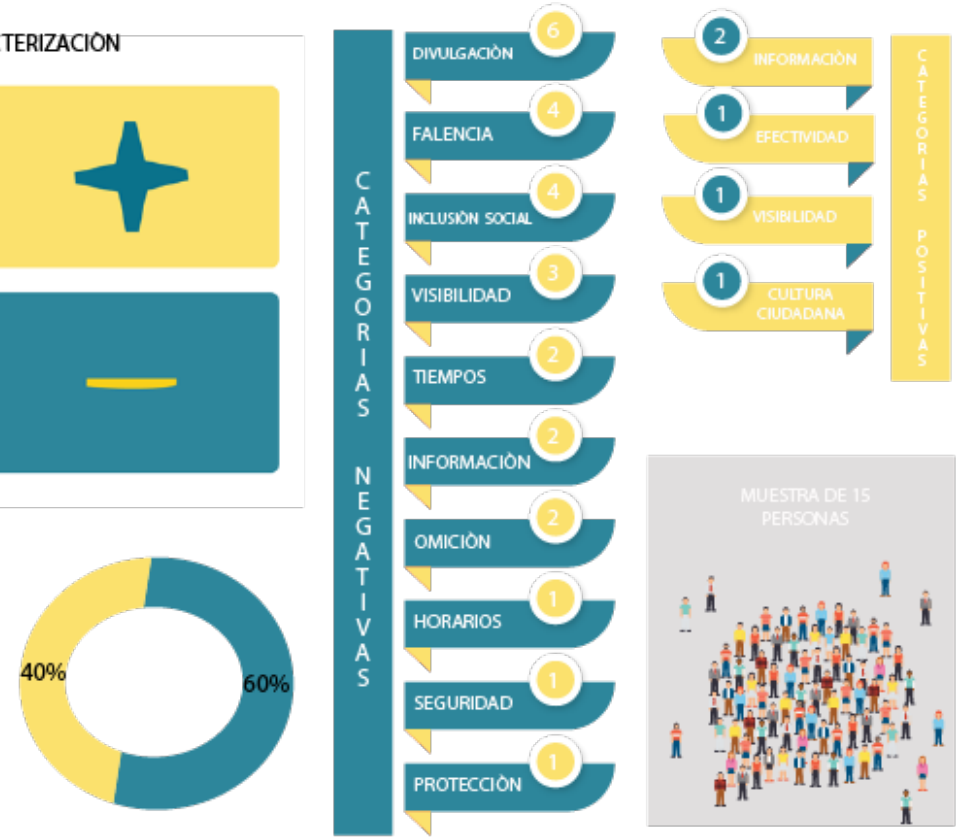

Figura 3. Grupo focal

Focus group. Se conformó con un grupo de 20 personas, entre ellas madres comunitarias, estudiantes de universidades y personas del común, provenientes de diferentes puntos de la ciudad. En primera medida se desarrolló una explicación del proyecto, se contextualizó la problemática que afronta el transporte público y, por último, se buscó la percepción de los usuarios al utilizar los paraderos implementados por Avante, por medio de la ficha de caracterización de aspectos negativos y aspectos positivos que observan las personas en las señales de información, obteniéndose como resultado un 60\% de aspectos negativos, entre ellos la falta de divulgación, falencias en la usabilidad, no presentan inclusión social y falta de visibilidad; y un $40 \%$ en aspectos positivos, como la importancia de la información de rutas, cultura ciudadana, efectividad y protección. Así mismo, por medio de un taller se pudo obtener las expectativas de un paradero óptimo para sus necesidades.

\section{- Segundo momento}

Pertinencia de la investigación en el escenario. Según el objeto de estudio, en el cual se basa el proyecto, en la movilidad urbana existe el organismo Avante-Sistema Integrado de Transporte Público (SITP) -Organización descentralizada del orden Municipal, que tiene por objetivo planear, coordinar, gestionar, desarrollar e implementar la movilidad -, ha logrado implementar el proyecto de movilidad en la ciudad de San Juan de Pasto, en el que se tienen en cuenta diferentes puntos para generar una correcta movilidad. 
La parte visual de señalética en los paraderos, fue creada y esta implementada desde el 28 de junio de 2017. Los elementos de información están dispuestos de manera vertical, y se los denomina paraderos tipo 1, la estructura es de aluminio y mide 3,38 metros de alto por 80 centímetros de ancho aproximadamente, dispone de una información en los paneles, la cual muestra las rutas. (Avante, 2002). Aproximadamente, son 700 señales situadas en toda la zona urbana y rural aledaña a Pasto; sin embargo, en la actualidad existe otro tipo de paraderos olvidados y en mal estado, alrededor de 7 en la zona urbana.
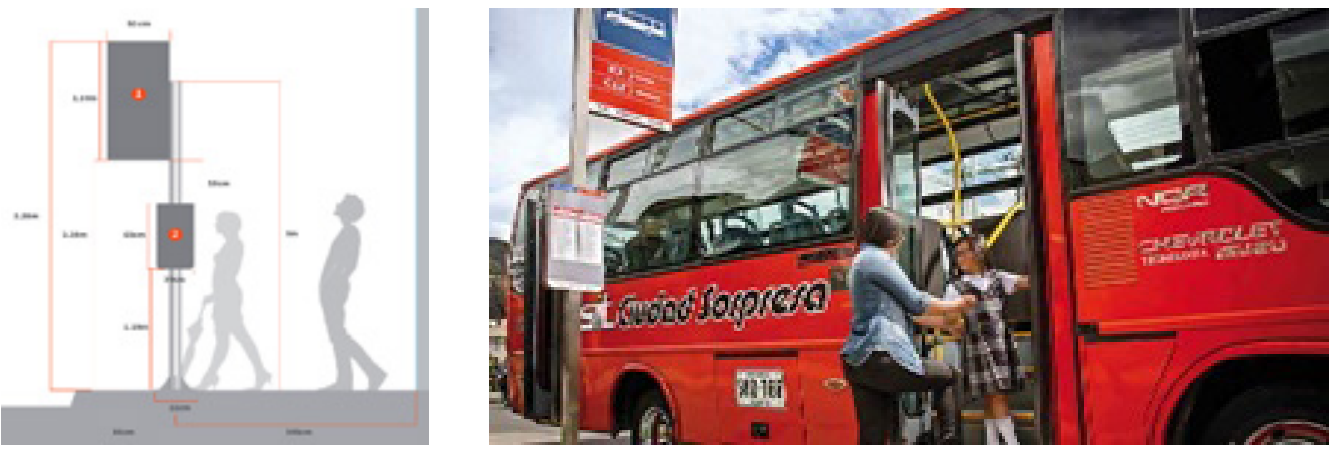

Figura 4. Señalética de paradero y vehículo del SITP-AVANTE 2017-2018 Fuente: Ilustración y fotografía del autor

Exploración del espacio en la ciudad. En este aspecto se recopilo información por medio de registros fotográficos en algunos puntos de la ciudad, que colaboraron para detectar algunos elementos implementados y cómo éstos ayudan a generar una dinámica en la movilidad urbana. Los resultados obtenidos en esta observación, fue de mobiliario urbano olvidado y en mal estado, situado en las zonas periféricas de Pasto: Parque Bolívar, Avenida Mijitayo, Barrio Lorenzo de Aldana, Barrios Sur Orientales y sector de la Universidad Mariana.

Por lo anterior, los elementos de movilidad no cumplen con su función, ya sea por su diseño, cultura ciudadana o por la mala ubicación en el espacio. Al respecto, se observó algo peculiar, cuando el individuo al detectar cierta necesidad de refugiarse, elabora un elemento improvisado en el barrio Lorenzo para ser más específicos, lo que da lugar a una oportunidad de diseño. 


\section{- Tercer momento}

\section{Lenguaje especifico.}

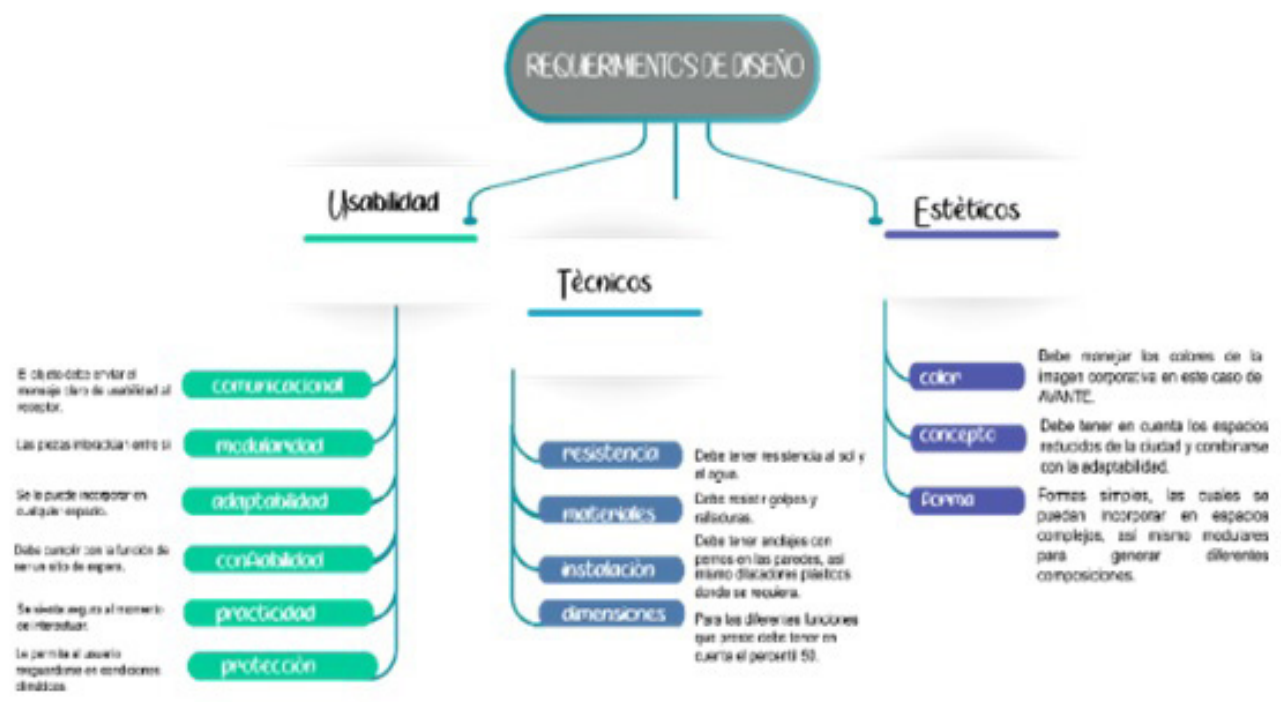

Figura 5. Mapa conceptual: 3 requerimientos de diseño y construcciòn de la investigación

Iteración. Es importante entender la iteración como: “La repetición de un acto para alcanzar un objetivo y suele retroalimentar un proceso" (Ballesteros, 2018, p.38). En la intención de alcanzar un diseño que se acople al trabajo de investigación, se tomó variables para llegar al objetivo, afianzando el conocimiento y, por ende, para darle valor a la investigación.

Para valorar la arquitectura colonial de la ciudad de Pasto, se realizó una observación de las formas y patrones que configuran las estructuras de los templos, tomando como objeto de estudio lo más representativo, como lo son: el Templo de San Juan Bautista, Templo de Cristo Rey Jesuitas, Iglesia de San Agustín y la Iglesia de San Sebastián, de los cuales se sintetizaron figuras geométricas que se repetían en la mayoría de los templos. Una vez realizado el estudio de las formas en $2 \mathrm{D}$, se procedió a la incorporación en estructuras 3D, para lo cual se utilizó el Diseño Paramétrico, método que se encarga de abstraer una idea o concepto, relacionado con los procesos geométricos y matemáticos, que permiten manipular con mayor precisión el diseño, para llegar a resultados óptimos (García, C. \& García Ríos, 2010).

Al tener un acercamiento con todas las variables, se procedió a realizar un acercamiento plástico, para descartar opciones y retroalimentar otras. En este proceso de llegar al diseño integral que abarque todos los componentes adecuados para un paradero en un espacio complejo, se construye otra variable para acercarse a lo que sería un artefacto convencional. 


\section{Concepto final: adaptabilidad}

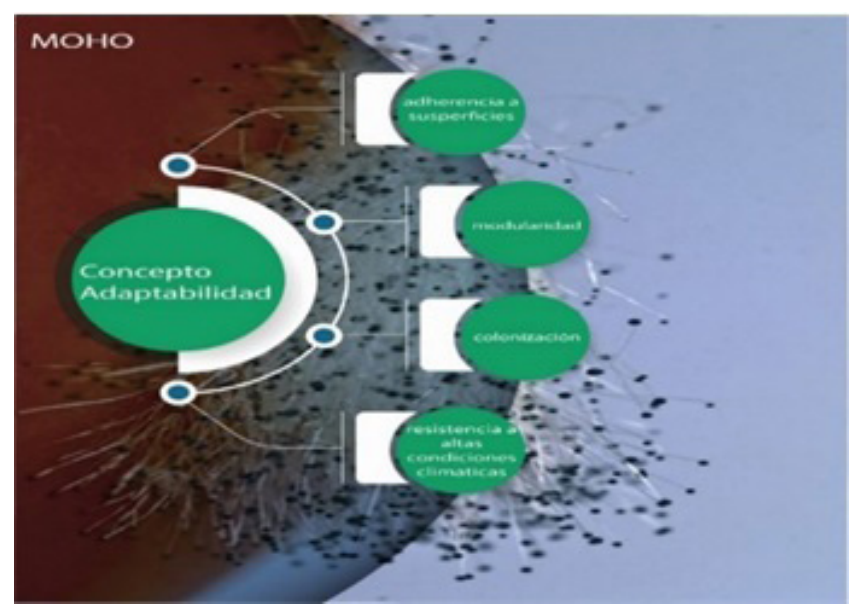

Figura 6. Mapa mental: 5 conceptos de adaptabilidad en la construcción de la investigación

Al analizar varios conceptos y retroalimentar algunas ideas, se llegó a la conclusión de que la adaptabilidad es clave para la elaboración del diseño que se quiere incorporar. Por esta razón, al partir de la naturaleza, se buscó la especie que mejor se adapte al medio, se pudo captar algo interesante que ocurría con el moho, ya que presenta características únicas, como la capacidad de adaptarse en condiciones climáticas extremas y su capacidad de expansión por medio de esporas, lo que le permite colonizar varios lugares:

Existe en la naturaleza el reino fungí que es encargado de clasificar la variedad de hongos, sin duda uno de los más interesantes es el moho ya que tienen ciertas características únicas, el moho es un hongo que se encarga de la descomposición de los alimentos, naciendo por un lugar del alimento y expandiéndose hasta cubrirlo. (Redfield, 2003, p.2)

La capacidad de adaptarse a las condiciones climáticas, genera gran adaptabilidad en situaciones extremas; por otra parte, la manera de expandirse por el terreno que posee, lo convierte en una fuente de adaptabilidad muy buena. Con base en su función mas no en su forma, se decidió escoger este tipo de elemento como concepto, ya que cumple con los requerimientos necesarios para formar un diseño adaptable en condiciones complejas y modular para poder colonizar varios espacios reducidos, y crear un objeto para el transporte público, en el que el objeto se establecerá como módulo, e, igual, se cumplirá con algunas necesidades de los usuarios, percibidas anteriormente. 


\section{Resultados}

\section{- Morfología de las ideas}

Ideación en maquetas: Módulos, Modelación 3D

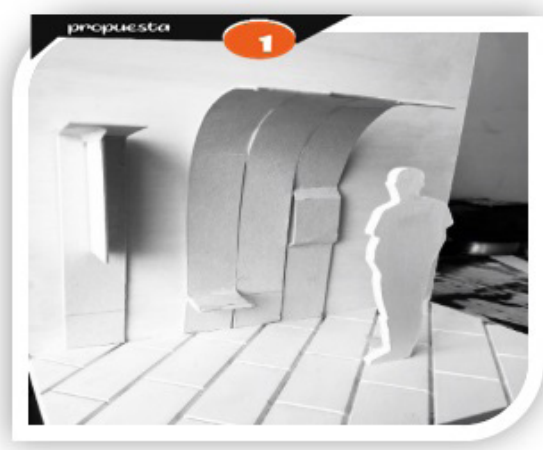

Figura 7. Propuesta

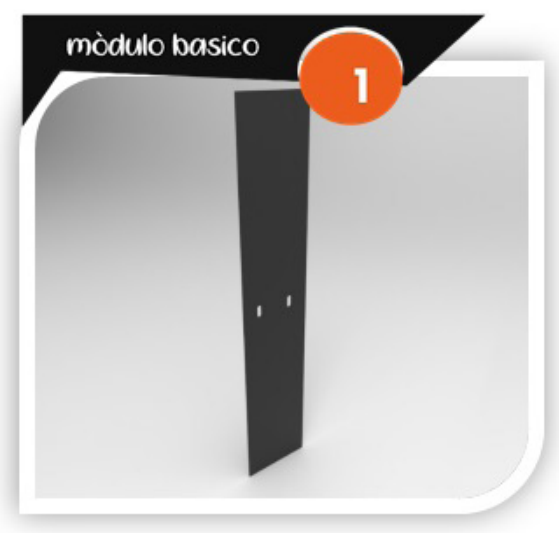

Figura 8. Módulo básico

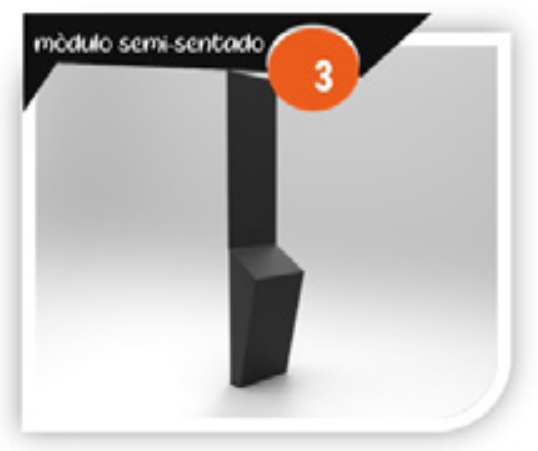

Figura 10. Módulo semisentado

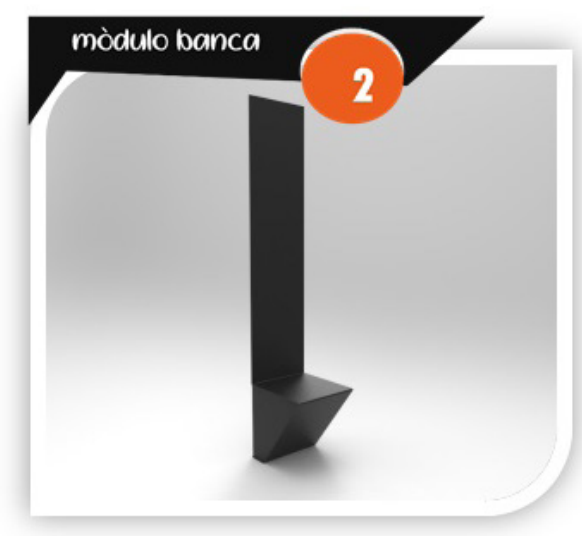

Figura 9. Banca

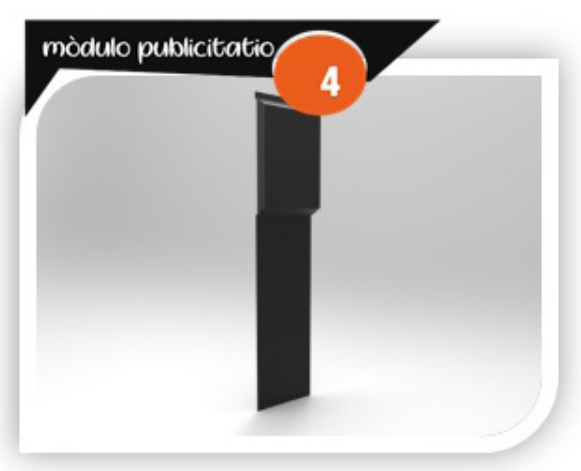

Figura 11. Módulo publicitario 


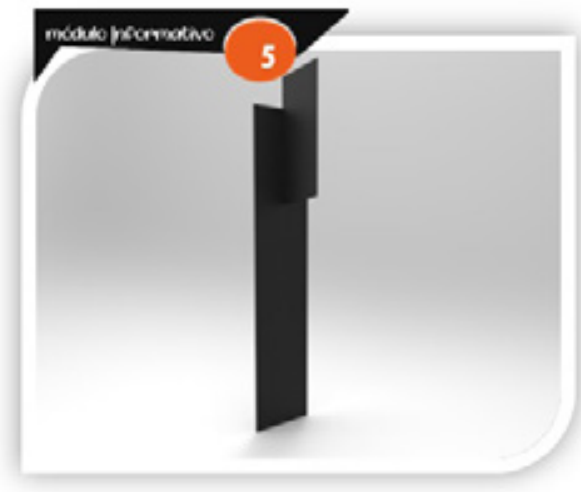

Figura 12. Módulo informativo

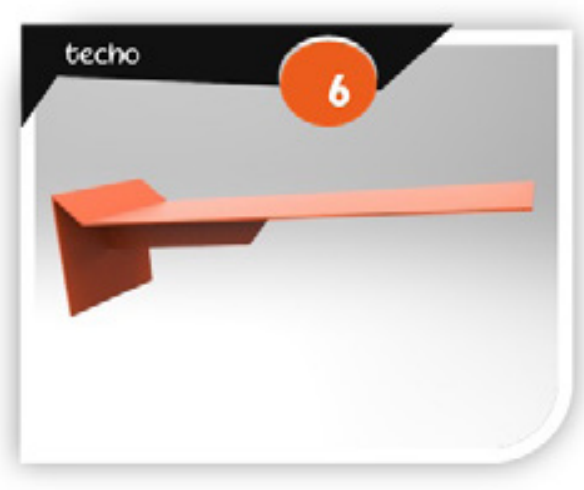

Figura 13. Techo

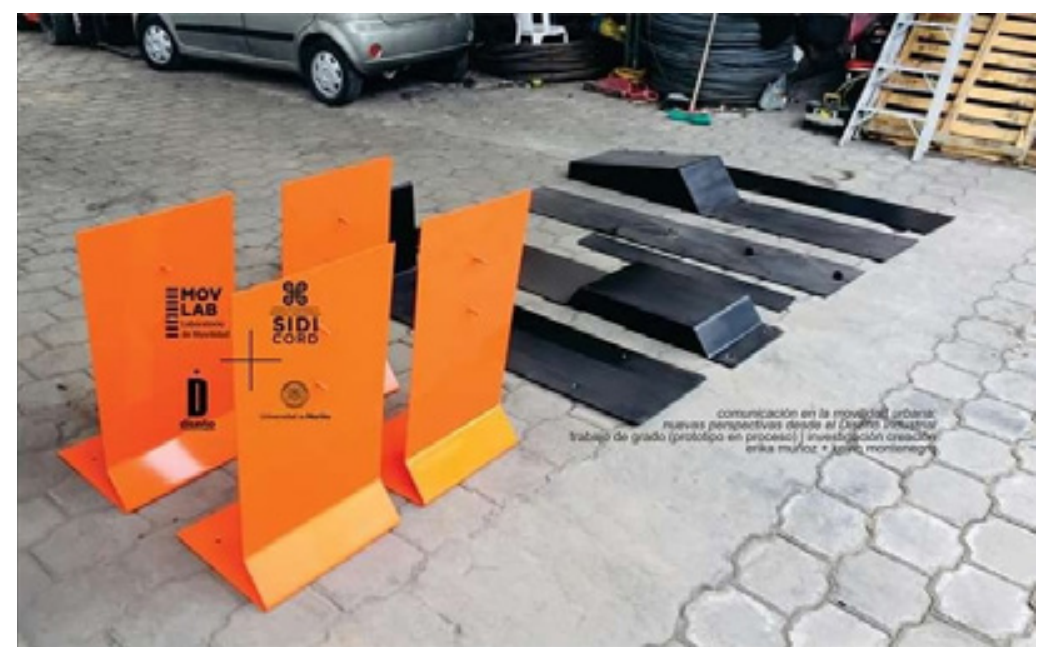

Figura 13. . Fabricación de los módulos

\section{Conclusiones}

Al analizar varios conceptos y retroalimentar algunas ideas, se llegó a la conclusión de que la adaptabilidad es clave para la elaboración del diseño que se quiere incorporar, siempre y cuando se cumpla con los requerimientos necesarios para formar un diseño adaptable en condiciones complejas y modulares para poder colonizar varios espacios reducidos, y crear un objeto para el transporte público, en el que el objeto se establecerá como módulo; e igual, se cumplirá con las necesidades de los usuarios.

Se establece, entonces, que los tipos de paraderos complementarios, en la ciudad de Pasto, es más conveniente disponerlos de forma vertical, ya que, de esta manera, resultan menos invasivos en la vía del peatón. Éstos, a su vez, serían incorporados en los muros para poder adaptarse al medio y, así, ofrecer al usuario comodidad y protección frente a los tiempos de espera del servicio de transporte. 
El proyecto no pretende disminuir el transporte informal ya que éste es un fenómeno que se presenta por la falta de oportunidades laborales en el Departamento de Nariño, pero si va enfocado a ofrecer una mejor calidad en el servicio de transporte público para los usuarios.

\section{Referencias}

Avante. (junio, 2002). Avante-Sistema Estrategico de Trasnporte Público [Mensaje en sitio web]. Recuperado de https://Setpasto.Gov.Co/Administracion/Nosotros/QuienesSomos

Avante. (19 de abril de 2017). Avante-Sistema Estrategico de Trasnporte Pùblico [Mensaje en sitio web]. Recuperado de https://Setpasto.Gov.Co/Administracion/PlanMaestro-De-Movilidad

Ballesteros, M. (2018). Investigar creando? Una guia para la investigación-creación en la academia. Bogota, DC.: Universidad del Bosque.

Departamento Administrativo Nacional de Estadística (DANE). (2018). Proyecciones de Población. Bogota, DC. Recuperado de https://Www.Dane.Gov.Co/

Departamento Administrativo Nacional de Estadística (DANE). (2020). Tasa de desempleo. Bogota, DC. Recuperado de https://Www.Dane.Gov.Co/

García, C. \& García Ríos, I. (2010). El diseño paramétrico y la geometría de las formas arquitectónicas. Recuperado de http://oa.upm.es/7664/1/INVE_MEM_2010_80494.pdf

Plan de Ordenamiento Territorial (POT). (2015). Pasto territorio con sentido, cuaderno diagnóstico fisico espacial (Tomo 1). Pasto: Alcaldía del Municipio de Pasto. Recuperado de http://Www.Sdp.Gov.Co/Sites/Default/Files/4-Documento-TecnicoDe-Soporte/_Libro\%201\%20dts.Pdf

Redfield, R. (agosto, 2003). Los mohos en el medio ambiente. Departament of Health and Human Services. Recuperado de https://www.cdc.gov/Mold/Es/Pdfs/Faqs.Pdf

Registro Unico Nacional de Transito (RUNT). (2019). Registro Unico Nacional de Transito. Bogotá, DC.: Conseción Runt.Sa. Recuperado de http://81494.Asset.Goto-9.Net/ Web_Public_Shared/Attached/81494/Estado_Revision_Tecnico_Mecanica_Por_ Departamentos.Pdf?Utm_Source=Email_Marketing\&Utm_Admin=81494\&Utm_ Medium=Email\&Utm_Campaign=EI_De_Los_Vehculos_Involucrados_En_ Accidentes_De_Trnsito_No_Tena

Ruíz, M. H. (2018). Evolución y diagnóstico del sistema de transporte público en Pasto. En Autor (Ed.), Evolución y diagnóstico del sistema de transporte público en Pasto (pp. 41-42). Bogotá, DC.: Universidad Santo Tomas.

Secretaría de Tránsito y Transporte del Municipio de Pasto. (2019). El Sistema Estratégico de Transporte Público de Pasto cuenta con más de 700 señales verticales de paraderos. Pasto: Alcaldia del Municipio de Pasto. Recuperado de https://Www. Pasto.Gov.Co/Index.Php/Transito-Y-Transporte/13196-El-Sistema-Estrategico-DeTransporte-Publico-De-Pasto-Cuenta-Con-Mas-De-700-Senales-Verticales-DeParaderos 


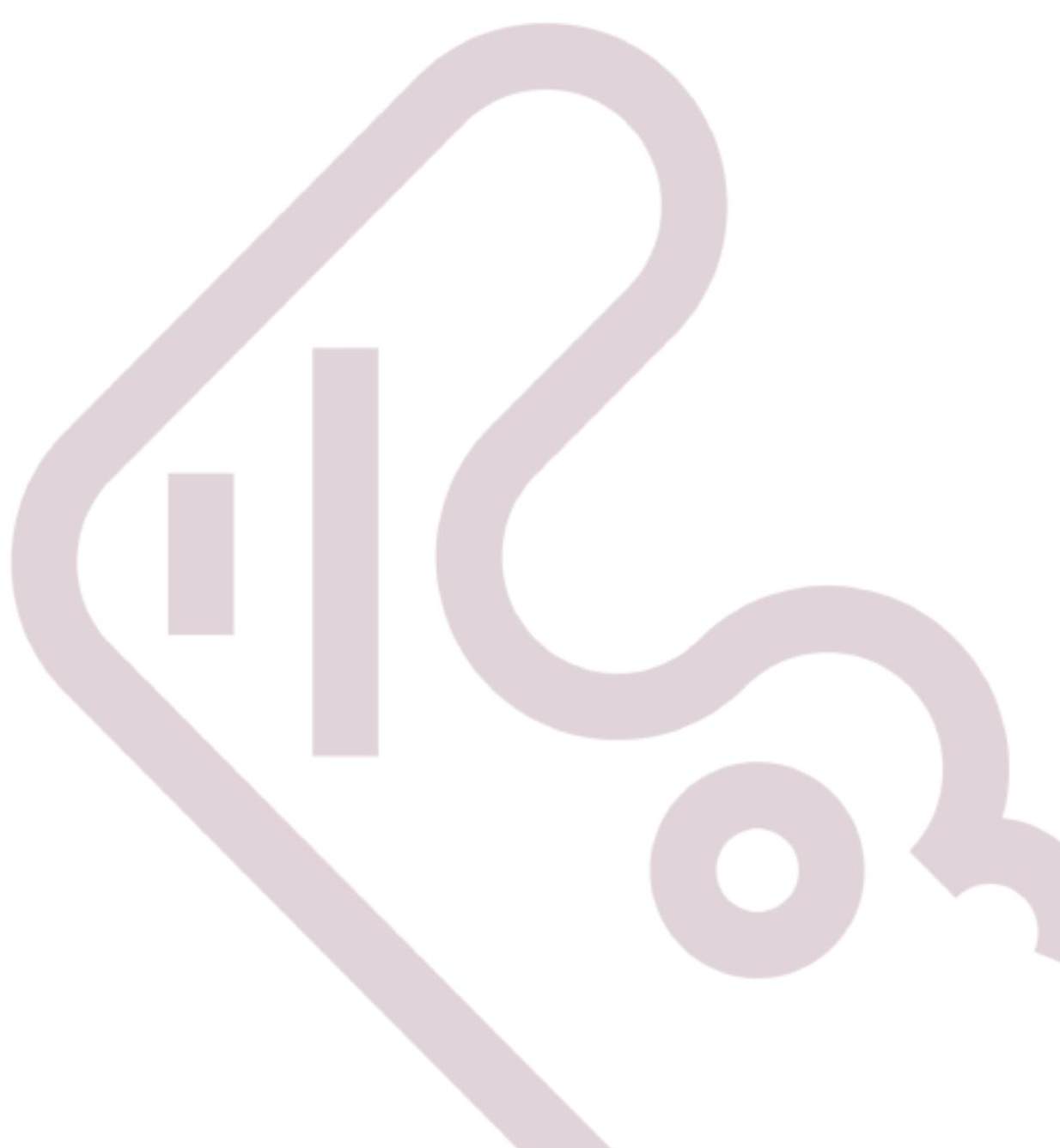

